\title{
Melatonin and Multiple Sclerosis: An Outline on Current Evidences
}

\section{Seyed-Hossein Abtahi ${ }^{1,3}$, Zahra Nasr $^{1,3}$, Niloofaralsadat Nourian ${ }^{4}$, Seyed-Mojtaba Abtahi ${ }^{6}$, Fatemeh Abrishamchi $^{5}$, Masoud Etemadifar ${ }^{1}$ and Mahboobeh Fereidan-Esfahani ${ }^{1,2}$}

${ }^{1}$ Isfahan Research Committee of Multiple Sclerosis (IRCOMS), Isfahan University of Medical Sciences, Isfahan, Iran

${ }^{2}$ Persia Research Center, Sady hospital, Isfahan, Iran

${ }^{3}$ Medical Students' Research Center, Isfahan University of Medical Sciences, Isfahan, Iran

${ }^{4}$ Medical School, Islamic Azad University, Najafabad Branch, Najafabad, Iran

${ }^{5}$ Department of Neurology, Medical School, Isfahan University of Medical Sciences, Isfahan, Iran

${ }^{6}$ Department of Otolaryngology/Head and Neck Surgery, School of Medicine, Isfahan University of Medical Sciences, Isfahan, Iran

\begin{abstract}
To date, in the literature, there have been a number of studies regarding the effects of Melatonin on MS course and pathogenesis; herein, we wish to bring a concise outline on such evidences to the readership.
\end{abstract}

\section{Keywords: Melatonin; Multiple sclerosis; Sleep; Vitamin D}

Multiple sclerosis (MS) is a complex demyelinating disorder resulting from multiplicity genetic and environmental risk factors. The substantial rise in MS prevalence and changing in the epidemiological pattern of MS have been shown in several population-based studies [1].

To date, in the literature, there have been a number of studies regarding the effects of Melatonin on MS course and pathogenesis; herein, we wish to bring a concise outline on such evidences to the readership.

Melatonin is mainly produced by pineal gland in dark phase and metabolized to principal metabolite 6-hydroxy-melatonin in the liver. Although, melatonin secretion has a constant rhythmic amplitude in each individual, significant differences has been shown among the general population [2].

Varied factors such as, high oxygen utilization; high concentration of polyunsaturated fatty acids; low concentrations of cytosolic antioxidants; and, existence of transition metals such as iron involved in the generation of hydroxyl radicals make the brain susceptible to radical damage [3]. On one hand, some theories for the role of oxidative stress, autoimmunity and inflammatory process have been proposed in the pathogenesis of MS lesions. On the other hand, the anti-inflammatory, immunomodulatory and antioxidative effects of melatonin have been previously well established [4]; theoretically, these protective effects could play an important role in MS pathogenesis, course and complications such as fatigue [5].

Some previous studies showed that the level of melatonin or its metabolites decreases in MS patients compared with normal controls and inversely might be correlated with MS disease activity [6,7]. Such findings could have been explained by the anti-inflammatory property of melatonin.

Complications such as sleep restriction and depression are common in MS. Melatonin secretion is directly affected by environmental stimuli such as light and in turn, regulates the sleep circle in humans. A recent study showed significant decrease in melatonin levels among patients with sleep restriction [6]. Relatively, increased risk of MS has also been shown in young patients with shift work [8]. Moreover, one of the routine treatments of MS patients i.e. Interferon-beta may result in increased level of serum melatonin, though, this medication was not shown to improve the sleep efficacy. Such results might have been due to other factors along with melatonin which could play a role in sleep disturbances; e.g. urinary complications; spasticity and muscle cramps; fatigue; depression; disease activity; and, location of CNS lesions.
In some reports, depression -- another common complication of MS -- has been associated with low nighttime level of serum melatonin [9]; a finding which can be speculatively explained by melatonin dysregulation in MS. However, some other studies [10,11] do not support such a linkage; this could be due to other confounding factors e.g. antidepressant agents, beta blockers or hormonal drugs, as well as age, light exposure and season.

Impressive geographical gradient with significantly higher incidence of MS in increasing latitude led scientists to evaluate the role of vitamin D in MS patients and also its relation with melatonin secretion [12,13]. Recently, Golan et al. [14] found that melatonin level is inversely related to $25-\mathrm{OH}-\mathrm{D}$ level. They adhered to the notion that '25-OH-D can bring a 'message of light' to the pineal gland and consequently decrease melatonin synthesis". Such possible interconnectivities between vitamin $\mathrm{D}$ and melatonin are an engaging issue to be opened up in future works. Further clinical and experimental studies should consider the concurrent role of melatonin and vitamin D on MS-related symptoms and disease course.

\section{References}

1. Etemadifar M, Abtahi SH, Akbari M, Murray RT, Ramagopalan SV, et al. (2013) Multiple sclerosis in Isfahan, Iran: an update. Mult Scler.

2. Lin GJ, Huang SH, Chen SJ, Wang CH, Chang DM, et al. (2013) Modulation by melatonin of the pathogenesis of inflammatory autoimmune diseases. Int $\mathrm{J}$ Mol Sci 14: 11742-11766.

3. Szczepanik M (2007) Melatonin and its influence on immune system. J Physio Pharmacol 58: 115-124.

4. Pajovic SB, Saicic ZS, Spasic MB, Petrovic VM (2003) The effect of ovarian hormones on antioxidant enzyme activities in the brain of male rats. Physiological research/Academia ScientiarumBohemoslovaca 52: 189-194.

5. Etemadifar M, Sayahi F, Abtahi SH, Shemshaki H, Dorooshi GA, et al. (2013)

*Corresponding author: Mahboobeh Fereidan-Esfahani, Isfahan MS Society, No.2, Behesht Building, Bozorgmehr Ave., Isfahan, Iran, Tel: +98.9380283952; E-mail:mf.esfahani@yahoo.com

Received December 13, 2013; Accepted January 30, 2014; Published February 15,2014

Citation: Abtahi SH, Nasr Z, Nourian N, Abtahi SM, Abrishamchi F, et al. (2014) Melatonin and Multiple Sclerosis: An Outline on Current Evidences. J Neurol Neurophysiol S12: 008. doi:10.4172/2155-9562.S12-008

Copyright: ( 2014 Abtahi SH, et al. This is an open-access article distributed under the terms of the Creative Commons Attribution License, which permits unrestricted use, distribution, and reproduction in any medium, provided the original author and source are credited. 
Citation: Abtahi SH, Nasr Z, Nourian N, Abtahi SM, Abrishamchi F, et al. (2014) Melatonin and Multiple Sclerosis: An Outline on Current Evidences. J Neurol Neurophysiol S12: 008. doi:10.4172/2155-9562.S12-008

Ginseng in the treatment of fatigue in multiple sclerosis: a randomized, placebocontrolled, double-blind pilot study. Int J Neurosci 123: 480-486.

6. Melamud L, Golan D, Luboshitzky R, Lavi I, Miller A (2012) Melatonin dysregulation, sleep disturbances and fatigue in multiple sclerosis. J Neurol Sci 314: $37-40$

7. Sandyk R (1993) Multiple sclerosis: the role of puberty and the pineal gland in its pathogenesis. Int J Neurosci 68: 209-225.

8. Hedström AK, Åkerstedt T, Hillert J, Olsson T, Alfredsson L (2011) Shift work at young age is associated with increased risk for multiple sclerosis. Ann Neurol 70: 733-741.

9. Akpinar Z, Tokgöz S, Gökbel H, Okudan N, Uğuz F, et al. (2008) The association of nocturnal serum melatonin levels with major depression in patients with acute multiple sclerosis. Psychiatry Res 161: 253-257.

10. Rubin RT, Heist EK, McGeoy SS, Hanada K, Lesser IM (1992) Neuroendocrine aspects of primary endogenous depression. XI. Serum melatonin measures in patients and matched control subjects. Archives of general psychiatry 49 558-567.

11. Sekula LK, Lucke JF, Heist EK, Czambel RK, Rubin RT (1997) Neuroendocrine aspects of primary endogenous depression. XV: Mathematical modeling of nocturnal melatonin secretion in major depressives and normal controls. Psychiatry research 69: 143-153.

12. Etemadifar M, Sajjadi S, Nasr Z, Firoozeei TS, Abtahi SH, et al. (2013) Epidemiology of multiple sclerosis in Iran: a systematic review. Eur Neurol 70 356-363.

13. Etemadifar M, Abtahi SH, Razmjoo H, Abtahi MA, Dehghani A et al. (2012) 25-hydroxyvitamin D Concentrations in Patients with Optic Neuritis as a Clinically Isolated Syndrome and Healthy Controls. Int J Prev Med 3: 313-317.

14. Golan D, Staun-Ram E, Glass-Marmor L, Lavi I, Rozenberg O, et al. (2013) The influence of vitamin D supplementation on melatonin status in patients with multiple sclerosis. Brain, behavior, and immunity 32: 180-185. 